\title{
Synthesis of novel triazolo[4,3-a]quinoxaline darivatives containing pyridinyl and thiazole nucleous
}

\author{
Haresh G. Kathrotiya, Yogesh T. Naliapara* \\ Department of Chemistry, Saurashtra University, Rajkot-360005, Gujarat, India \\ *E-mail address: naliaparachem@yahoo.co.in
}

Keywords: [1,2,4]triazolo[4,3-a]quinoxaline; Pyridinyl; Thiazole; lodobenzene diacetate

\begin{abstract}
On pursuing research about $[1,2,4]$ triazolo[4,3-a]quinoxaline, in this paper we report a small library of novel class of $[1,2,4]$ triazolo[4,3-a]quinoxaline derivatives containing pyridinyl thiazole moiety. Particularly valuable features of this method include high yield, broad substrate scope, shorter reaction time and straightforward procedure. The structures of new compounds have been characterized on the basis of elemental analysis, FT- IR, ${ }^{1} \mathrm{H}$ NMR, ${ }^{13} \mathrm{C} \mathrm{NMR}$, and mass spectral data.
\end{abstract}

\section{INTRODUCTION}

Nitrogen containing heterocyclic compounds are present in all the living beings where these performs important biological functions. Nitrogenous heterocyclic compounds occurred widely in roast food and drugs and posses different pharmacological properties due to oxidation of nitrogen in molecule [1]. 1,2,4-Triazole motif is found in a number of chemotherapeutic agent such as conazole fungicides, triazolobenzodiazepine, rizatriptan and ribavirin, that find a wide array of biological activities such as anti-fungal [2], antibacterial [3], anticonvulsant [4] and anticancer [5], analgesic [6], anti-inflammatory [7], antiviral [8], insecticide [9], antidepressant [10]. Also quinoxaline derivatives possess a broad spectrum of biological activities including anti-bacterial, anti-viral, antiinflammatory, anticancer, and kinase inhibitors [11]. In addition, quinoxaline derivatives have been evaluated as anthelmintic agents, semiconductors, dyes and biocides [12, 13].

There are many methods for the synthesis of triazolo quinoxalines which are shown here. Reaction of 1-ethyl-3-hydrazinylquinoxalin-2(1H)-one with carben disulphide and potassium hydroxide formed triazolo quinoxalines [14]. Cupric acetate in acetic acid, cyclised (E)-2benzylidene-1-(2-chloroquinoxalin-3-yl)hydrazine to triazolo quinoxaline derivatives [15]. A double site- and regio-selective 1,3-dipolar cycloaddition of arylnitrilimines to quinoxalines gave bis-1,2,4-triazolo[4,3-a:3',4'-c] quinoxalines [16]. N'-(5-methyl-2 nitrophenyl)benzohydrazide reacted with ethyl (chlorocarbonyl)formate to give triazolo quinoxalines [17]. The solvent free reaction of 3-methylquinoxalin-2-ylhydrazones and iodobenzene diacetate afforded triazolo quinoxalines with good yield [18]. Here we report the synthesis of novel triazolo quinoxaline darivatives containing pyridine and thiazole nucleous by using iodobenzene diacetate as catalyst. The constitutions of all the products were confirmed using ${ }^{1} \mathrm{H}$ NMR, ${ }^{13} \mathrm{C}$ NMR, FTIR, and elemental analysis.

\section{EXPERIMENTAL}

Required all reagents were obtained commercially. Solvents were purified and dried before being used. All melting points were taken in open capillaries and are uncorrected. Thin-layer chromatography (TLC, on aluminium plates precoated with silica gel, $60 \mathrm{~F}_{254}, 0.25 \mathrm{~mm}$ thickness) (Merck, Darmstadt, Germany) was used for monitoring the progress of all reactions, purity and homogeneity of the synthesized compounds; eluent-hexane:ethyl acetate: (2:8). UV radiation and/or iodine were used as the visualizing agents. Elemental analysis $(\% \mathrm{C}, \mathrm{H}, \mathrm{N})$ was carried out by Perkin-Elmer 2400 series-II elemental analyzer (Perkin-Elmer, USA) and all compounds are within $\pm 0.4 \%$ of theory specified. The IR spectra were recorded in $\mathrm{KBr}$ on a Perkin-Elmer Spectrum GX FT-IR Spectrophotometer (Perkin-Elmer, USA) and only the characteristic peaks are reported in 
$\mathrm{cm}^{-1} .{ }^{1} \mathrm{H}$ NMR and ${ }^{13} \mathrm{C}$ NMR spectra were recorded in DMSO- $d_{6}$ on a Bruker Avance $400 \mathrm{~F}(\mathrm{MHz})$ spectrometer (Bruker Scientific Corporation Ltd., Switzerland) using solvent peak as internal standard at $400 \mathrm{MHz}$ and $100 \mathrm{MHz}$ respectively. Chemical shifts are reported in parts per million (ppm). Mass spectra were scanned on a Shimadzu LCMS 2010 spectrometer (Shimadzu, Tokyo, Japan).

\subsection{Method for the synthesis of compound (1a)}

To a suspension of substituted benzene-1,2-diamine $(5 \mathrm{mmol})$, diethyl oxalate $(5 \mathrm{mmol})$ in ethanol $(10 \mathrm{ml}), 1 \mathrm{ml}$ of pyrrolidine was added and the reaction mixture was refluxed for $4 \mathrm{~h}$. On completion of reaction, monitored by TLC, the the separated substituted quinoxaline- $2,3(1 H, 4 H)$ dione was filtered and washed with ethanol and dried. Further mixture of appropriate substituted quinoxaline-2,3(1H,4H)-dione $(5 \mathrm{gm})$ and $3 \mathrm{ml}$ of DMF in $\mathrm{POCl}_{3}(25 \mathrm{ml})$ were charged in $100 \mathrm{ml}$ round bottom flask equipped with condenser. The reaction mixture was stirred at reflux for $4 \mathrm{~h}$. On completion of reaction, monitored by TLC, The reaction mixture was poured in to chilled water. The separated solid was filtered, washed well with water and dried to get pure substituted 2,3dichloroquinoxaline.

\subsection{Method for the synthesis of compound (3c)}

To a suspension of substituted 2,3-dichloroquinoxaline $1 \mathbf{a}(5 \mathrm{mmol})$ and 4-(pyridin-4yl)thiazol-2-amine $\mathbf{2 b}(5 \mathrm{mmol})$ in IPA $(10 \mathrm{ml})$, dry potassium carbonate $(5 \mathrm{mmol})$ was added and the reaction mixture was refluxed for $3 \mathrm{~h}$. After completion of the reaction, the reaction mixture was cooled to room temperature and poured in cold water. The separated solid was filtered, washed with water, dried and recrystallized from ethanol to afford analytically pure substituted 3-chloro- $N$-(4(pyridin-4-yl)thiazol-2-yl)quinoxalin-2-amine.

\subsection{General method for the synthesis of compounds 4(a-t)}

To a suspension of substituted 3-chloro- $N$-(4-(thiophen-2-yl)thiazol-2-yl)quinoxalin-2-amine $3 \mathbf{c}(5 \mathrm{mmol})$ and hydrazine hydrate $(5 \mathrm{mmol})$ in methanol was refluxed for $2 \mathrm{~h}$. After completion of the reaction(monitored by TLC), cooled the content and filter the separated solid, dried in oven to furnished analytically pure substituted 3-hydrazinyl- $N$-(4-(thiophen-2-yl)thiazol-2-yl)quinoxalin-2amine. Then took substituted 3-hydrazinyl- $N$-(4-(thiophen-2-yl)thiazol-2-yl)quinoxalin-2-amine (5 $\mathrm{mmol})$ and various aromatic aldehydes $(5 \mathrm{mmol})$ in methanol, refluxed for $2 \mathrm{~h}$. After completion of the reaction(monitored by TLC), cooled the content and filter the separated solid, dried in oven to furnished analytically pure 3-((E)-2-benzylidenehydrazinyl)- $N$-(4-(thiophen-2-yl)thiazol-2yl)quinoxalin-2-amine derivatives. Then Iodobenzene diacetate $(15 \mathrm{mmol})$ was added to a solution of 3-((E)-2-benzylidenehydrazinyl)- $N$-(4-(thiophen-2-yl)thiazol-2-yl)quinoxalin-2-amine derivatives $(5 \mathrm{mmol})$ in chloroform $(10 \mathrm{ml})$ withine one minitue and stirred the solution for 40-50 minitues at rt. After the completion of reaction, monitored by the TLC, the mixture was washed with water followed by saturated $\mathrm{NaHCO}_{3}$ solution. The organic phase was dried over anhydrous $\mathrm{Na}_{2} \mathrm{SO}_{4}$ and solvent was removed under reduced pressure to afford solid product which recrystallized from methanol to obtain pure compounds $\mathbf{4 a - 4 t}$. The physicochemical and spectral properties of all the newly synthesized compounds $\mathbf{4 a - 4 t}$. are presented below.

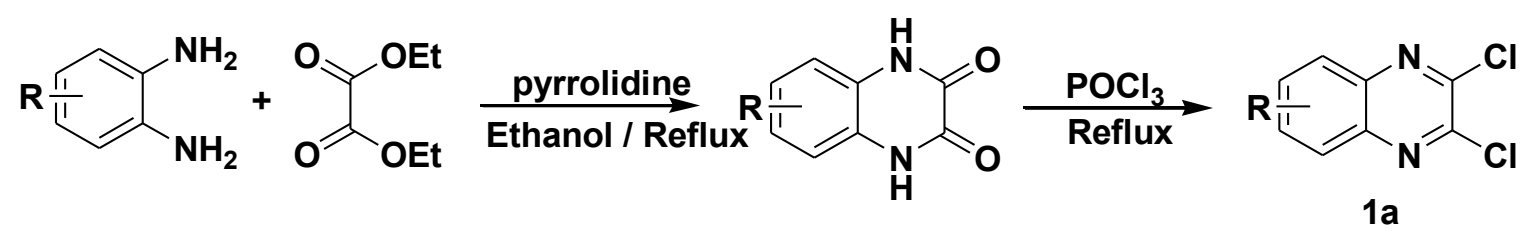

Where $\mathrm{R}=\mathrm{NO}_{2}, \mathrm{Br}, \mathrm{H}$

Scheme 1 Synthetic pathway for the synthesis of intermediate 1a 
<smiles>[R][Tl]1C=CC=c2nc(Cl)c(Cl)nc2=C1</smiles>

$1 \mathrm{a}$<smiles>Nc1nc(-c2ccncc2)cs1</smiles>

Where $\mathrm{R}=\mathrm{NO}_{2}, \mathrm{Br}, \mathrm{H}$<smiles>[R][X]1C=CC=c2nc(Nc3nc(-c4ccncc4)cs3)c(Cl)nc2=C1</smiles>

3c

Scheme 2 Synthetic pathway for the synthesis of intermediate $3 \mathrm{c}$<smiles>[R][X]1ccc2nc(Cl)c(Nc3nc(-c4ccncc4)cs3)nc2c1</smiles>

$3 c$<smiles>[R][C@H](N)[C@@H](N)OC</smiles><smiles>[R]CC=c1nc(Nc2nc(-c3ccncc3)cs2)c(NN)nc1=CCC[R]</smiles>

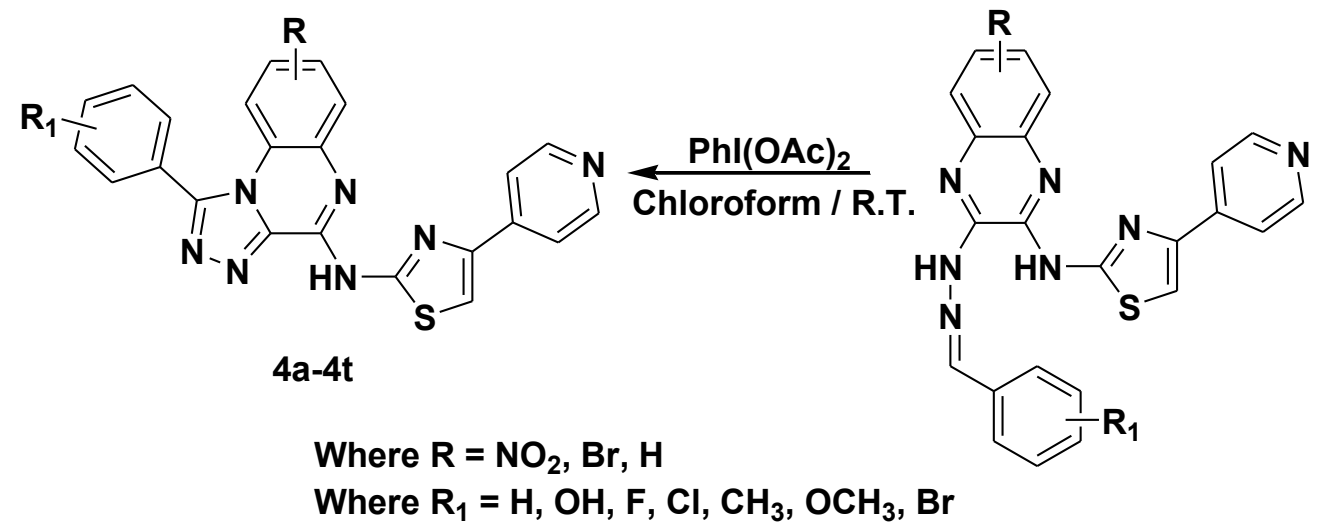

Scheme 3 Synthetic pathway for the synthesis of tiazolo quinoxalines $4 \mathrm{a}-4 \mathrm{t}$ 
Tabel-1 Synthesis of triazolo quinoxalines

\begin{tabular}{|c|c|c|c|c|c|}
\hline Entry & $\mathbf{R}$ & $\mathbf{R}_{\mathbf{1}}$ & $\mathbf{R T ( m i n . )}$ & Yield \% & $\mathbf{m p}^{\mathbf{0}} \mathbf{C}$ \\
\hline $4 \mathrm{a}$ & $\mathrm{NO}_{2}$ & $\mathrm{H}$ & 42 & 88 & $150-152$ \\
\hline $4 \mathrm{~b}$ & $\mathrm{NO}_{2}$ & $4-\mathrm{OH}$ & 43 & 82 & $162-164$ \\
\hline $4 \mathrm{c}$ & $\mathrm{NO}_{2}$ & $4-\mathrm{F}$ & 45 & 80 & $153-155$ \\
\hline $4 \mathrm{~d}$ & $\mathrm{NO}_{2}$ & $4-\mathrm{Cl}$ & 48 & 76 & $167-169$ \\
\hline $4 \mathrm{e}$ & $\mathrm{NO}_{2}$ & $4-\mathrm{CH}_{3}$ & 43 & 82 & $182-184$ \\
\hline $4 \mathrm{f}$ & $\mathrm{NO}_{2}$ & $4-\mathrm{OCH}_{3}$ & 42 & 85 & $176-178$ \\
\hline $4 \mathrm{~g}$ & $\mathrm{NO}_{2}$ & $4-\mathrm{Br}$ & 41 & 77 & $181-183$ \\
\hline $4 \mathrm{~h}$ & $\mathrm{Br}$ & $\mathrm{H}$ & 42 & 81 & $159-161$ \\
\hline $4 \mathrm{i}$ & $\mathrm{Br}$ & $4-\mathrm{OH}$ & 43 & 76 & $153-155$ \\
\hline $4 \mathrm{j}$ & $\mathrm{Br}$ & $4-\mathrm{F}$ & 44 & 73 & $149-151$ \\
\hline $4 \mathrm{k}$ & $\mathrm{Br}$ & $4-\mathrm{Cl}$ & 43 & 71 & $177-179$ \\
\hline $4 \mathrm{l}$ & $\mathrm{Br}$ & $4-\mathrm{CH}$ & 40 & 90 & $181-183$ \\
\hline $4 \mathrm{~m}$ & $\mathrm{Br}$ & $4-\mathrm{OCH}$ & 40 & 92 & $142-144$ \\
\hline $4 \mathrm{n}$ & $\mathrm{Br}$ & $4-\mathrm{Br}$ & 48 & 69 & $169-171$ \\
\hline $4 \mathrm{o}$ & $\mathrm{H}$ & $\mathrm{H}$ & 42 & 84 & $133-135$ \\
\hline $4 \mathrm{p}$ & $\mathrm{H}$ & $4-\mathrm{OH}$ & 43 & 79 & $143-145$ \\
\hline $4 \mathrm{q}$ & $\mathrm{H}$ & $4-\mathrm{F}$ & 43 & 81 & $148-150$ \\
\hline $4 \mathrm{r}$ & $\mathrm{H}$ & $4-\mathrm{Cl}$ & 45 & 78 & $172-174$ \\
\hline $4 \mathrm{~s}$ & $\mathrm{H}$ & $4-\mathrm{CH} H_{3}$ & 44 & 83 & $166-168$ \\
\hline $4 \mathrm{t}$ & $\mathrm{H}$ & $4-\mathrm{OCH}$ & 43 & 83 & $147-149$ \\
\hline
\end{tabular}



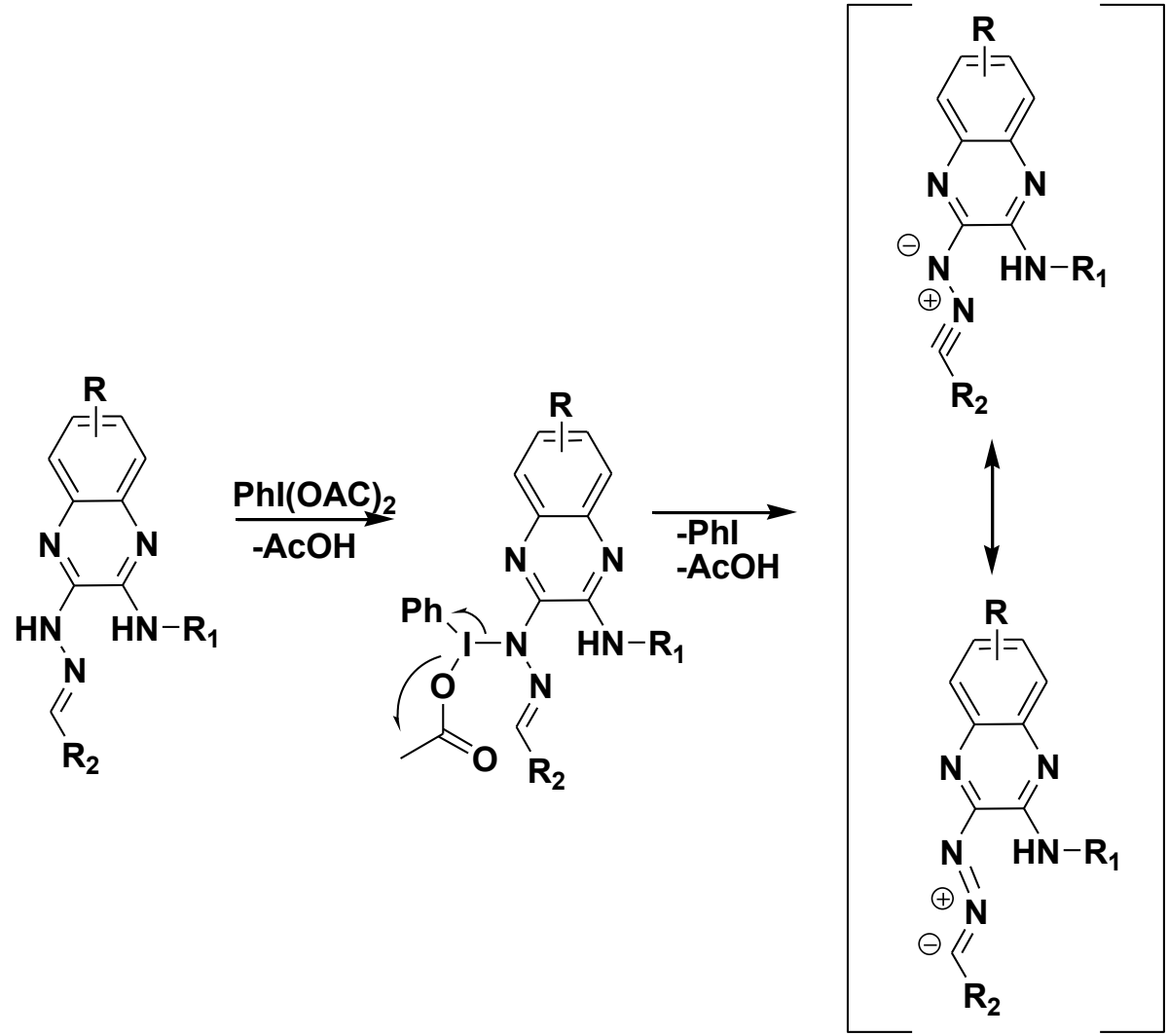
Where $\mathrm{R}=\mathrm{NO}_{2}, \mathrm{Br}, \mathrm{H}$ $\mathbf{R}_{1}=$ Heterocyles $\mathbf{R}_{\mathbf{2}}=$ Aryl

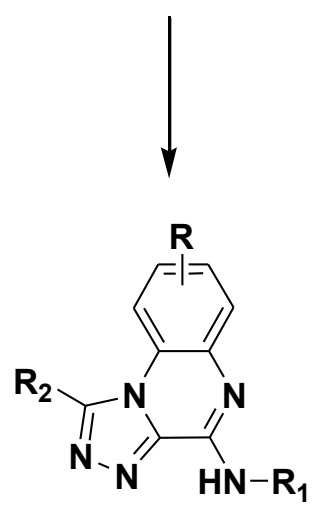

Scheme 4 Mechanism for the synthesis of tiazolo quinoxalines $4 a-4 t$

The required 4-(pyridin-4-yl)thiazol-2-amine $\mathbf{2 b}$ was prepared by solid phase reaction according to literature procedure [19].

In this work, a new series of triazolo quinoxaline derivatives $4 \mathbf{a}-4 \mathbf{t}$ have been synthesized by reaction of 3-((E)-2-benzylidenehydrazinyl)- $N$-(4-(thiophen-2-yl)thiazol-2-yl)quinoxalin-2-amine derivatives and iodobenzene diacetate as catalyst in chloroform at room temperature (Scheme 3). A possible mechanism for the reaction is outlined in (Scheme- 4). The reaction occurs via dehydrogenative cyclisation. The electrophilic attack of iodobenzene diacetate on 3-((E)-2benzylidenehydrazinyl)- $N$-(4-(thiophen-2-yl)thiazol-2-yl)quinoxalin-2-amine derivatives to generates the nitrile imine. Later on, due to the loss of iodobenzene and acetic acid, ring closure took place through quinoxaline ring nitrogen and leads to the formation of final compounds $\mathbf{4 a - 4 t}$.

The structures of the obtained compounds were fully characterized by ${ }^{1} \mathrm{H} \mathrm{NMR},{ }^{13} \mathrm{C} \mathrm{NMR}$ and FT-IR spectral data and molecular weight of some selected compounds confirmed by mass spectrometry. ${ }^{1} \mathrm{H}$ NMR (DMSO-d6) spectrum of compound $\mathbf{4 a}$ exhibited a singlet around d 10.33 ppm for $-\mathrm{NH}-$ proton and multiplets around 7.12-8.16 ppm for aromatic protons. The ${ }^{13} \mathrm{C}$ NMR 
spectrum of $\mathbf{4 a}$ is in good agreement with the structure assigned. The peaks at d 113.62-145.00 ppm are assigned to aromatic carbons. All compounds gave satisfactory elemental analyses. The mass spectra detected the expected molecular ion signals corresponding to respective molecular formula of synthesized compounds. Mass spectra of compound 4a gave molecular ion peak at $466.1(\mathrm{M}+1)$ corresponding to molecular formula $\mathrm{C}_{23} \mathrm{H}_{14} \mathrm{~N}_{8} \mathrm{O}_{\mathrm{s}} \mathrm{S}$. All spectroscopic data have been given in experimental section.

\section{SPECTRAL DATA}

\section{7-nitro-1-phenyl- $N$-(4-(pyridin-4-yl)thiazol-2-yl)-[1,2,4]triazolo[4,3-a]quinoxalin-4 amine (4a)}

light yellow solid, yield 88\%, m.p. 150-152 ${ }^{\circ} \mathrm{C}$, IR (KBr, v, $\left.\mathrm{cm}^{-1}\right): 3288$ (N-H Str.), 3021 (Ar C-H Str.), ${ }^{1} \mathrm{H}$ NMR (400 MHz, DMSO- $\left.d_{6}\right) \delta_{\mathrm{H}}(\mathrm{ppm}): \delta 7.120-8.166(\mathrm{~m}, 13 \mathrm{H}, \mathrm{Ar}-\mathrm{H}), 10.336(\mathrm{~s}, 1 \mathrm{H}, \mathrm{NH})$. ${ }^{13} \mathrm{C}$ NMR $\left(100 \mathrm{MHz}, \mathrm{DMSO}-d_{6}\right) \delta_{\mathrm{C}}(\mathrm{ppm}): 113.62,114.28,116.00,117.21,118.36,119.89,120.34$, $122.00,123.56,124.00,125.88,127.03,128.36,129.30,131.20,132.78,133.64,135.69,137.28$, 139.64, 140.12, 143.65, 145.00(Ar-C). $\mathrm{MS}\left(\mathrm{M}^{+}\right)$: 466.10, Anal. Calcd. for $\mathrm{C}_{23} \mathrm{H}_{14} \mathrm{~N}_{8} \mathrm{O}_{\mathrm{s}} \mathrm{S}$ (466.47): C 59.22, H 3.03, N 24.02 Found: C 59.18, H 3.15, N 24.18\%.

\section{4-(4-(4-(pyridin-4-yl)thiazol-2-ylamino)-7-nitro-[1,2,4]triazolo[4,3-a]quinoxalin-1-yl)phenol (4b)}

light yellow solid, yield 82\%, m.p. 162-164 C, IR (KBr, v, $\mathrm{cm}^{-1}$ ): 3216 (N-H Str.), 3033 (Ar C-H Str.), ${ }^{1} \mathrm{H}$ NMR (400 MHz, DMSO- $\left.d_{6}\right) \delta_{\mathrm{H}}(\mathrm{ppm}): \delta 4.980$ (s, 1H, OH), 6.713-7.698 (m, 12H, Ar-H), $10.456(\mathrm{~s}, 1 \mathrm{H}, \mathrm{NH}) .{ }^{13} \mathrm{C} \mathrm{NMR}\left(100 \mathrm{MHz}, \mathrm{DMSO}-d_{6}\right) \delta_{\mathrm{C}}(\mathrm{ppm}): 112.12,113.20,115.01,117.3$, $118.66,119.79,120.64,122.88,123.38,124.02$, 125.18, 127.33, 128.11, 129.31, 131.23, 132.68, 133.55, 135.79, 136.18, 137.14, 140.18, 143.70, 145.02 (Ar-C). $M S\left(\mathrm{M}^{+}\right)$: 482.09, Anal. Calcd. for $\mathrm{C}_{23} \mathrm{H}_{14} \mathrm{~N}_{8} \mathrm{O}_{3} \mathrm{~S}$ (482.47): C 57.26, H 2.92, N 23.22 Found: C 57.49, H 2.80, N 23.44\%.

\section{1-(4-fluorophenyl)-7-nitro- $N$-(4-(pyridin-4-yl)thiazol-2-yl)-[1,2,4]triazolo[4,3-a]quinoxalin-4- amine (4c)}

light yellow solid, yield 80\%, m.p. $153-155^{\circ} \mathrm{C}$, IR (KBr, v, cm $\left.{ }^{-1}\right): 3266$ (N-H Str.), 3041 (Ar C-H Str.), ${ }^{1} \mathrm{H}$ NMR (400 MHz, DMSO- $\left.d_{6}\right) \delta_{\mathrm{H}}$ (ppm): $\delta 6.980-7.846$ (m, 12H, Ar-H), 10.663 (s, 1H, NH). ${ }^{13} \mathrm{C}$ NMR (100 MHz, DMSO- $\left.d_{6}\right) \delta_{\mathrm{C}}(\mathrm{ppm}): 113.25,114.63,116.45,117.23,117.96,119.79,121.36$, $122.01,123.86,124.50,125.70,126.05,127.66,129.50,130.21,132.68,133.22,135.70,137.18$, 138.24, 141.13, 143.55, 145.16 (Ar-C). $M S\left(M^{+}\right)$: 484.09, Anal. Calcd. for $\mathrm{C}_{23} \mathrm{H}_{13} \mathrm{FN}_{8} \mathrm{O}_{2} \mathrm{~S}(484.47)$ : C 57.02, H 2.70, N 23.13 Found: C 57.36, H 2.66, N 23.17\%.

\section{1-(4-chlorophenyl)-7-nitro- $N$-(4-(pyridin-4-yl)thiazol-2-yl)-[1,2,4]triazolo[4,3-a]quinoxalin-4- amine (4d)}

light yellow solid, yield 76\%, m.p. 167-169 $\mathrm{C}$, IR (KBr, v, cm ${ }^{-1}$ ): 3278 (N-H Str.), 3012 (Ar C-H Str.), ${ }^{1} \mathrm{H}$ NMR (400 MHz, DMSO- $\left.d_{6}\right) \delta_{\mathrm{H}}(\mathrm{ppm}): \delta$ 7.123-8.200 (m, 12H, Ar-H), $10.656(\mathrm{~s}, 1 \mathrm{H}$, $\mathrm{NH}) .{ }^{13} \mathrm{C}$ NMR $\left(100 \mathrm{MHz}, \mathrm{DMSO}-d_{6}\right) \delta_{\mathrm{C}}(\mathrm{ppm}): 108.36,109.36,110.22,111.36,112.40,113.56$, $115.21,116.80,117.36,118.90,120.22,121.45,122.00,124.38,126.39,127.00,128.94,131.00$, 132.65, 134.65, 136.48, 137.45, 139.45(Ar-C). $\mathrm{MS}\left(\mathrm{M}^{+}\right)$: 500.06, Anal. Calcd. for $\mathrm{C}_{23} \mathrm{H}_{13} \mathrm{ClN}_{8} \mathrm{O}_{2} \mathrm{~S}$ (500.92): C 55.15, H 2.62, N 22.37 Found: C 55.07, H 2.48, N 22.22\%.

\section{7-nitro- $N$-(4-(pyridin-4-yl)thiazol-2-yl)-1-p-tolyl-[1,2,4] triazolo[4,3-a]quinoxalin-4-amine (4e)}

light yellow solid, yield 82\%, m.p. 182-184 ${ }^{\circ} \mathrm{C}$, IR (KBr, v, $\mathrm{cm}^{-1}$ ): 3222 (N-H Str.), 3009 (Ar C-H Str.), ${ }^{1} \mathrm{H}$ NMR (400 MHz, DMSO- $\left.d_{6}\right) \delta_{\mathrm{H}}(\mathrm{ppm}): \delta 2.540$ (s, 3H, $\left.\mathrm{CH}_{3}\right), 6.880-7.986(\mathrm{~m}, 12 \mathrm{H}, \mathrm{Ar}-\mathrm{H})$, 
$10.320(\mathrm{~s}, 1 \mathrm{H}, \mathrm{NH}) .{ }^{13} \mathrm{C}$ NMR $\left(100 \mathrm{MHz}, \mathrm{DMSO}-d_{6}\right) \delta_{\mathrm{C}}(\mathrm{ppm}): 26.48\left(\mathrm{Ar}-\mathrm{CH}_{3}\right) .108 .96,109.78$, $110.33,112.11,112.90,113.16,114.23,115.81,116.31,117.91,119.30,121.25,122.06,124.18$, 126.31, 127.08, 128.84, 131.22, 132.15, 133.61, 135.40, 137.15, 139.11(Ar-C). MS( $\left.\mathrm{M}^{+}\right): 480.11$, Anal. Calcd. for $\mathrm{C}_{24} \mathrm{H}_{16} \mathrm{~N}_{8} \mathrm{O}_{2} \mathrm{~S}$ (480.50): C 59.99, $\mathrm{H}$ 3.36, N 23.32 Found: C 59.66, H 3.30, N $23.42 \%$.

\section{1-(4-methoxyphenyl)-7-nitro- $N$-(4-(pyridin-4-yl)thiazol-2-yl)-[1,2,4]triazolo[4,3-a]quinoxalin- 4-amine (4f)}

light yellow solid, yield 85\%, m.p. 176-178 C, IR (KBr, v, $\mathrm{cm}^{-1}$ ): 3265 (N-H Str.), 3041 (Ar C-H Str.), ${ }^{1} \mathrm{H}$ NMR (400 MHz, DMSO- $\left.d_{6}\right) \delta_{\mathrm{H}}(\mathrm{ppm}): \delta 3.828\left(\mathrm{~s}, 3 \mathrm{H}, \mathrm{OCH}_{3}\right), 7.125-8.222(\mathrm{~m}, 12 \mathrm{H}, \mathrm{Ar}-$ $\mathrm{H}), 10.630(\mathrm{~s}, 1 \mathrm{H}, \mathrm{NH}) .{ }^{13} \mathrm{C}$ NMR $\left(100 \mathrm{MHz}, \mathrm{DMSO}-d_{6}\right) \delta_{\mathrm{C}}(\mathrm{ppm}): 55.61\left(\mathrm{OCH}_{3}\right) .107 .25,108.36$, $110.12,111.36,112.57,113.58,114.90,116.51,117.88,119.10,120.36,122.34,123.87,125.39$, 126.50, 127.10, 129.64, 131.45, 133.00, 135.60, 137.28, 138.60, 139.20(Ar-C). MS(M $\left.{ }^{+}\right): 496.11$, Anal. Calcd. for $\mathrm{C}_{23} \mathrm{H}_{13} \mathrm{BrN}_{8} \mathrm{O}_{2} \mathrm{~S}$ (545.37): C 50.65, H 2.40, N 20.55 Found: C 50.45, H 2.65, N $20.66 \%$.

\section{1-(4-bromophenyl)-7-nitro- $N$-(4-(pyridin-4-yl)thiazol-2-yl)-[1,2,4]triazolo[4,3-a]quinoxalin-4- amine (4g)}

light yellow solid, yield 87\%, m.p. 181-183 ${ }^{\circ} \mathrm{C}$, IR (KBr, v, $\mathrm{cm}^{-1}$ ): 3298 (N-H Str.), 3049 (Ar C-H Str.), ${ }^{1} \mathrm{H}$ NMR (400 MHz, DMSO- $\left.d_{6}\right) \delta_{\mathrm{H}}(\mathrm{ppm}): \delta$ 7.120-8.312 (m, 12H, Ar-H), $10.686(\mathrm{~s}, 1 \mathrm{H}$, $\mathrm{NH}) .{ }^{13} \mathrm{C}$ NMR $\left(100 \mathrm{MHz}, \mathrm{DMSO}-d_{6}\right) \delta_{\mathrm{C}}(\mathrm{ppm}): 107.33,108.22,110.11,112.00,112.70,113.18$, $114.20,116.51,117.18,118.10,119.96,120.35,123.66,125.69,126.55,127.33,129.24,131.55$, 133.01, 135.10, 136.24, 137.61, 138.21(Ar-C). $\mathrm{MS}\left(\mathrm{M}^{+}\right)$: 544.01, Anal. Calcd. for $\mathrm{C}_{23} \mathrm{H}_{13} \mathrm{BrN}_{8} \mathrm{O}_{2} \mathrm{~S}$ (545.37): C 50.65, H 2.40, N 20.55 Found: C 50.45, H 2.65, N 20.66\%.

\section{7-bromo-1-phenyl- $N$-(4-(pyridin-4-yl)thiazol-2-yl)-[1,2,4]triazolo[4,3-a]quinoxalin-4-amine} (4h)

light yellow solid, yield 81\%, m.p. 159-161 ${ }^{\circ} \mathrm{C}$, IR (KBr, v, $\mathrm{cm}^{-1}$ ): 3255 (N-H Str.), 3025 (Ar C-H Str.), ${ }^{1}$ H NMR (400 MHz, DMSO- $\left.d_{6}\right) \delta_{\mathrm{H}}(\mathrm{ppm}): \delta 6.974-7.856(\mathrm{~m}, 13 \mathrm{H}, \mathrm{Ar}-\mathrm{H}), 10.333(\mathrm{~s}, 1 \mathrm{H}, \mathrm{NH})$. ${ }^{13} \mathrm{C}$ NMR (100 MHz, DMSO- $\left.d_{6}\right) \delta_{\mathrm{C}}(\mathrm{ppm}): 108.21,109.26,111.17,112.30,112.87,113.66,114.88$, $116.74,117.60,119.20,120.63,122.25,124.36,125.19,126.70,127.12,129.24,130.25,132.01$, 134.50, 136.20, 137.10, 138.27(Ar-C). $\mathrm{MS}\left(\mathrm{M}^{+}\right)$: 499.02, Anal. Calcd. for $\mathrm{C}_{23} \mathrm{H}_{14} \mathrm{BrN}_{7} \mathrm{~S}$ (500.37): C 55.21, H 2.82, N 19.59 Found: C 55.18, H 2.70, N 19.42\%.

\section{4-(4-(4-(pyridin-4-yl)thiazol-2-ylamino)-7-bromo-[1,2,4] triazolo[4,3-a]quinoxalin-1-yl)phenol} (4i)

light yellow solid, yield 76\%, m.p. $153-155^{\circ} \mathrm{C}$, IR (KBr, v, $\left.\mathrm{cm}^{-1}\right)$ : 3226 (N-H Str.), 3044 (Ar C-H Str.), ${ }^{1} \mathrm{H}$ NMR (400 MHz, DMSO- $\left.d_{6}\right) \delta_{\mathrm{H}}(\mathrm{ppm}): \delta 4.916(\mathrm{~s}, 1 \mathrm{H}, \mathrm{OH}), 7.156-8.200(\mathrm{~m}, 12 \mathrm{H}, \mathrm{Ar}-\mathrm{H})$, $10.226(\mathrm{~s}, 1 \mathrm{H}, \mathrm{NH}) .{ }^{13} \mathrm{C} \mathrm{NMR}\left(100 \mathrm{MHz}, \mathrm{DMSO}-d_{6}\right) \delta_{\mathrm{C}}(\mathrm{ppm}): 112.36,114.20,115.40,116.34$, $117.80,118.65,119.01,120.22,121.36,124.56,126.57,127.80,129.40,131.24,133.00,135.60$, 136.00, 138.08, 139.56, 141.20, 142.00, 143.36, 144.60(Ar-C). $M S\left(\mathrm{M}^{+}\right)$: 515.02, Anal. Calcd. for $\mathrm{C}_{23} \mathrm{H}_{13} \mathrm{BrFN}_{7} \mathrm{~S}$ (518.36): C 53.29, H 2.53, N 18.91 Found: C 53.14, H 2.68, N 18.80\%.

7-bromo-1-(4-fluorophenyl)- $N$-(4-(pyridin-4-yl)thiazol-2-yl)-[1,2,4]triazolo[4,3-a]quinoxalin-4amine (4j)

light yellow solid, yield 73\%, m.p. $149-151^{\circ} \mathrm{C}$, IR (KBr, v, $\left.\mathrm{cm}^{-1}\right)$ : 3268 (N-H Str.), 3018 (Ar C-H Str.), ${ }^{1} \mathrm{H}$ NMR (400 MHz, DMSO- $\left.d_{6}\right) \delta_{\mathrm{H}}(\mathrm{ppm}): \delta$ 7.110-8.245 (m, 12H, Ar-H), 10.665 (s, 1H, NH). 
${ }^{13} \mathrm{C}$ NMR $\left(100 \mathrm{MHz}, \mathrm{DMSO}-d_{6}\right) \delta_{\mathrm{C}}(\mathrm{ppm}): 112.46,114.11,115.26,116.45,117.10,118.35,119.36$, $120.55,122.16,123.46,126.80,127.50,129.30,131.29,133.01,134.28,136.25,137.05,138.16$, 141.21, 142.26, 143.33, 144.20 (Ar-C). $M S\left(M^{+}\right)$: 517.01, Anal. Calcd. for $\mathrm{C}_{23} \mathrm{H}_{13} \mathrm{BrFN}_{7} \mathrm{~S}(518.36)$ : C 53.29, H 2.53, N 18.91 Found: C 53.14, H 2.68, N 18.80\%.

7-bromo-1-(4-chlorophenyl)- $N$-(4-(pyridin-4-yl)thiazol-2-yl)-[1,2,4]triazolo[4,3-a]quinoxalin4-amine (4k)

light yellow solid, yield 71\%, m.p. 177-179 C, IR (KBr, v, $\mathrm{cm}^{-1}$ ): 3301 (N-H Str.), 3028 (Ar C-H Str.), ${ }^{1} \mathrm{H}$ NMR $\left(400 \mathrm{MHz}, \mathrm{DMSO}-d_{6}\right) \delta_{\mathrm{H}}(\mathrm{ppm}): \delta 6.715-7.856(\mathrm{~m}, 12 \mathrm{H}, \mathrm{Ar}-\mathrm{H}), 10.213(\mathrm{~s}, 1 \mathrm{H}$, $\mathrm{NH}) .{ }^{13} \mathrm{C}$ NMR $\left(100 \mathrm{MHz}, \mathrm{DMSO}-d_{6}\right) \delta_{\mathrm{C}}(\mathrm{ppm}): 113.20,114.23,116.20,117.21,118.36,119.32$, $121.00,123.36,125.18,126.54,127.00,128.69,130.22,132.05,134.56,136.20,137.16,139.20$, 141.20, 143.00, 144.21, 145.22, 146.90(Ar-C). $\mathrm{MS}\left(\mathrm{M}^{+}\right)$: 532.98, Anal. Calcd. for $\mathrm{C}_{23} \mathrm{H}_{13} \mathrm{BrClN}_{7} \mathrm{~S}$ (534.82): C 51.65, H 2.45, N 18.33 Found: C 51.42, H 2.51, N 18.51\%.

7-bromo- $N$-(4-(pyridin-4-yl)thiazol-2-yl)-1-p-tolyl-[1,2,4]triazolo[4,3-a]quinoxalin-4-amine (4I)

light yellow solid, yield 90\%, m.p. 181-183 ${ }^{\circ} \mathrm{C}$, IR (KBr, v, $\mathrm{cm}^{-1}$ ): 3292 (N-H Str.), 3016 (Ar C-H Str.), ${ }^{1} \mathrm{H}$ NMR (400 MHz, DMSO- $\left.d_{6}\right) \delta_{\mathrm{H}}(\mathrm{ppm}): \delta 2.563\left(\mathrm{~s}, 3 \mathrm{H}, \mathrm{CH}_{3}\right), 6.876-7.888(\mathrm{~m}, 12 \mathrm{H}, \mathrm{Ar}-$ $\mathrm{H}), 10.415(\mathrm{~s}, 1 \mathrm{H}, \mathrm{NH}) .{ }^{13} \mathrm{C}$ NMR $\left(100 \mathrm{MHz}, \mathrm{DMSO}-d_{6}\right) \delta_{\mathrm{C}}(\mathrm{ppm}): 24.78\left(\mathrm{Ar}-\mathrm{CH}_{3}\right), 113.18$, $114.36,116.78,117.80,118.22$, 119.12, 120.00, 122.16, 124.30, 125.14, 127.01, 128.89, 130.12, 132.22, 133.16, 135.21, 137.36, 138.21, 140.22, 142.03, 143.11, 144.21, 145.80(Ar-C). MS(M $\left.{ }^{+}\right)$: 513.04, Anal. Calcd. for $\mathrm{C}_{24} \mathrm{H}_{16} \mathrm{BrN}_{7} \mathrm{~S}$ (514.40): C 56.04, H 3.14, N 19.06 Found: C 56.32, H 3.51, N $19.46 \%$.

7-bromo-1-(4-methoxyphenyl)- $N$-(4-(pyridin-4-yl)thiazol-2-yl)-[1,2,4]triazolo[4,3-a]quinoxalin -4-amine(4m)

light yellow solid, yield 92\%, m.p. 142-144 C, IR (KBr, v, $\mathrm{cm}^{-1}$ ): 3305 (N-H Str.), 3035 (Ar C-H Str.), ${ }^{1} \mathrm{H}$ NMR $\left(400 \mathrm{MHz}, \mathrm{DMSO}-d_{6}\right) \delta_{\mathrm{H}}(\mathrm{ppm}): \delta 4.002\left(\mathrm{~s}, 3 \mathrm{H}, \mathrm{OCH}_{3}\right), 7.230-8.300(\mathrm{~m}, 12 \mathrm{H}, \mathrm{Ar}-$ $\mathrm{H}), 10.412(\mathrm{~s}, 1 \mathrm{H}, \mathrm{NH}) .{ }^{13} \mathrm{C}$ NMR $\left(100 \mathrm{MHz}, \mathrm{DMSO}-d_{6}\right) \delta_{\mathrm{C}}(\mathrm{ppm}): 56.38\left(\mathrm{OCH}_{3}\right), 112.21,113.21$, $115.21,116.22$, 117.26, 118.31, 121.05, 123.22, 125.46, 126.34, 127.23, 128.64, 130.18, 132.18, 134.95, 135.21, 136.26, 137.29, 140.10, 142.10, 144.20, 145.12, 147.00(Ar-C). $\mathrm{MS}\left(\mathrm{M}^{+}\right): 529.03$, Anal. Calcd. for $\mathrm{C}_{24} \mathrm{H}_{16} \mathrm{BrN}_{7} \mathrm{OS}$ (530.40): C 54.35, H 3.04, N 18.49 Found: C 54.17, H 3.12, N $18.61 \%$.

\section{7-bromo-1-(4-bromophenyl)- $N$-(4-(pyridin-4-yl)thiazol-2-yl)-[1,2,4]triazolo[4,3-a]quinoxalin- 4-amine (4n)}

light yellow solid, yield 69\%, m.p. $169-171^{\circ} \mathrm{C}$, IR (KBr, v, $\left.\mathrm{cm}^{-1}\right)$ : 3241 (N-H Str.), 3008 (Ar C-H Str.), ${ }^{1} \mathrm{H}$ NMR $\left(400 \mathrm{MHz}, \mathrm{DMSO}-d_{6}\right) \delta_{\mathrm{H}}(\mathrm{ppm}): \delta 7.123-8.236(\mathrm{~m}, 12 \mathrm{H}, \mathrm{Ar}-\mathrm{H}), 10.500(\mathrm{~s}, 1 \mathrm{H}$, $\mathrm{NH}) .{ }^{13} \mathrm{C}$ NMR $\left(100 \mathrm{MHz}, \mathrm{DMSO}-d_{6}\right) \delta_{\mathrm{C}}(\mathrm{ppm}): 110.32,112.36,113.24,114.50,116.20,117.18$, $118.32,120.18,122.34,124.56,127.18,129.00,131.41,132.00,135.44,136.18,137.19,139.34$, 142.13, 143.00, 144.08, 145.12, 146.00(Ar-C). $\mathrm{MS}\left(\mathrm{M}^{+}\right)$: 576.93, Anal. Calcd. for $\mathrm{C}_{23} \mathrm{H}_{13} \mathrm{Br}_{2} \mathrm{~N}_{7} \mathrm{~S}$ (579.27): C 47.69, H 2.26, N 16.93 Found: C 47.61, H 2.82, N 16.85\%.

\section{1-phenyl- $N$-(4-(pyridin-4-yl)thiazol-2-yl)-[1,2,4]triazolo[4,3-a]quinoxalin-4-amine (40)}

light yellow solid, yield 84\%, m.p. 133-135 C, IR (KBr, v, cm ${ }^{-1}$ ): 3268 (N-H Str.), 3036 (Ar C-H Str.), ${ }^{1} \mathrm{H}$ NMR (400 MHz, DMSO- $\left.d_{6}\right) \delta_{\mathrm{H}}$ (ppm): $\delta$ 7.200-8.100 (m, 14H, Ar-H), 10.456 (s, 1H, NH). ${ }^{13} \mathrm{C}$ NMR $\left(100 \mathrm{MHz}, \mathrm{DMSO}-d_{6}\right) \delta_{\mathrm{C}}(\mathrm{ppm}): 110.82,112.23,113.25,114.66,115.26,117.30,118.12$, 
$120.78,122.14,124.36,127.22,129.01,130.43,131.08,133.42,135.28,136.29,138.94,140.14$, 142.00, 144.01, 145.62, 146.20 (Ar-C). $\mathrm{MS}\left(\mathrm{M}^{+}\right)$: 421.11, Anal. Calcd. for $\mathrm{C}_{23} \mathrm{H}_{15} \mathrm{~N}_{7} \mathrm{~S}$ (421.48): C 65.54, H 3.59, N 23.26 Found: C 65.34, H 3.42, N 23.50\%.

\section{4-(4-(4-(pyridin-4-yl)thiazol-2-ylamino)-[1,2,4]triazolo[4,3-a]quinoxalin-1-yl)phenol (4p)}

light yellow solid, yield 79\%, m.p. 143-145 C, IR (KBr, v, $\mathrm{cm}^{-1}$ ): 3251 (N-H Str.), 3018 (Ar C-H Str.), ${ }^{1} \mathrm{H}$ NMR (400 MHz, DMSO- $\left.d_{6}\right) \delta_{\mathrm{H}}(\mathrm{ppm}): \delta 4.908$ (s, $\left.1 \mathrm{H}, \mathrm{OH}\right), 6.778-7.898$ (m, 13H, Ar-H), $10.313(\mathrm{~s}, 1 \mathrm{H}, \mathrm{NH}) .{ }^{13} \mathrm{C}$ NMR $\left(100 \mathrm{MHz}, \mathrm{DMSO}-d_{6}\right) \delta_{\mathrm{C}}(\mathrm{ppm}): 105.36,107.40,108.37,109.90$, $111.00,113.56,114.25,115.30,117.34,118.38,119.00,121.08,122.00,123.45,125.36,127.30$, 129.88, 131.45, 133.00, 135.46, 137.88, 139.01, 141.23(Ar-C). $\mathrm{MS}\left(\mathrm{M}^{+}\right)$: 437.11, Anal. Calcd. for $\mathrm{C}_{23} \mathrm{H}_{15} \mathrm{~N}_{7} \mathrm{OS}$ (437.48): C 63.15, H 3.46, N 22.41 Found: C 63.23, H 3.68, N 22.32\%.

\section{1-(4-fluorophenyl)- $N$-(4-(pyridin-4-yl)thiazol-2-yl)-[1,2,4]triazolo[4,3-a]quinoxalin-4-amine(4q)}

light yellow solid, yield 81\%, m.p. 148-150 C, IR (KBr, v, $\mathrm{cm}^{-1}$ ): 3274 (N-H Str.), 3029 (Ar C-H Str.), ${ }^{1} \mathrm{H}$ NMR (400 MHz, DMSO- $\left.d_{6}\right) \delta_{\mathrm{H}}(\mathrm{ppm}): \delta$ 7.120-8.210 (m, 13H, Ar-H), $10.226(\mathrm{~s}, 1 \mathrm{H}, \mathrm{NH})$. ${ }^{13} \mathrm{C}$ NMR (100 MHz, DMSO- $\left.d_{6}\right) \delta_{\mathrm{C}}(\mathrm{ppm}): 105.22,107.83,108.22,109.34,110.10,112.16,113.20$, $114.39,115.30,117.88,119.02,121.11,122.85,123.44,124.30,126.31,129.19,131.22,133.78$, 135.40, 136.80, 138.51, 140.21 (Ar-C). $M S\left(M^{+}\right)$: 439.10, Anal. Calcd. for $\mathrm{C}_{23} \mathrm{H}_{14} \mathrm{FN}_{7} \mathrm{~S}$ (439.47): C 62.86, H 3.21, N 22.31 Found: C 62.70, H 3.32, N 22.42\%.

\section{1-(4-chlorophenyl)- $N$-(4-(pyridin-4-yl)thiazol-2-yl)-[1,2,4]triazolo[4,3-a]quinoxalin-4-amine(4r)}

light yellow solid, yield 78\%, m.p. 172-174 C, IR (KBr, v, $\mathrm{cm}^{-1}$ ): 3280 (N-H Str.), 3022 (Ar C-H Str.), ${ }^{1} \mathrm{H}$ NMR (400 MHz, DMSO- $\left.d_{6}\right) \delta_{\mathrm{H}}$ (ppm): $\delta 6.978-7.800(\mathrm{~m}, 13 \mathrm{H}, \mathrm{Ar}-\mathrm{H}), 10.236(\mathrm{~s}, 1 \mathrm{H}, \mathrm{NH})$. ${ }^{13} \mathrm{C}$ NMR (100 MHz, DMSO- $\left.d_{6}\right) \delta_{\mathrm{C}}(\mathrm{ppm}): 107.16,108.40,109.35,110.90,111.01,113.56,114.33$, $115.22,116.30,117.18,119.10,121.12,122.05,123.65,125.06,128.10,129.85,130.15,132.05$, 134.16, 136.80, 137.08, 139.20 (Ar-C). $\mathrm{MS}\left(\mathrm{M}^{+}\right)$: 455.07, Anal. Calcd. for $\mathrm{C}_{23} \mathrm{H}_{14} \mathrm{ClN}_{7} \mathrm{~S}$ (455.92): $\mathrm{C}$ 60.59, H 3.10, N 21.51 Found: C 60.72, H 3.44, N 21.32\%.

\section{$N$-(4-(pyridin-4-yl)thiazol-2-yl)-1-p-tolyl-[1,2,4]triazolo[4,3-a]quinoxalin-4-amine (4s)}

light yellow solid, yield 83\%, m.p. 166-168 $\mathrm{C}$, IR (KBr, v, $\mathrm{cm}^{-1}$ ): 3328 (N-H Str.), 3033 (Ar C-H Str.), ${ }^{1} \mathrm{H}$ NMR (400 MHz, DMSO- $\left.d_{6}\right) \delta_{\mathrm{H}}(\mathrm{ppm}): \delta 2.658$ (s, 3H, $\left.\mathrm{CH}_{3}\right), 6.900-7.894(\mathrm{~m}, 13 \mathrm{H}, \mathrm{Ar}-\mathrm{H})$, $10.456(\mathrm{~s}, 1 \mathrm{H}, \mathrm{NH}) .{ }^{13} \mathrm{C}$ NMR $\left(100 \mathrm{MHz}, \mathrm{DMSO}-d_{6}\right) \delta_{\mathrm{C}}(\mathrm{ppm}): 23.50\left(\mathrm{Ar}-\mathrm{CH}_{3}\right), 112.20,113.60$, $114.20,116.02,117.18,119.80,120.22,122.00,124.36,125.39,126.07,127.35,128.25,130.25$, $131.49,132.25,133.00,135.60,137.80,139.20,140.01,142.22,143.00(A r-C) . M S\left(\mathrm{M}^{+}\right): 435.13$, Anal. Calcd. for $\mathrm{C}_{24} \mathrm{H}_{17} \mathrm{~N}_{7} \mathrm{~S}$ (435.50): C 66.19, H 3.93, N 22.51 Found: C 66.72, H 3.88, N 22.41\%.

\section{1-(4-methoxyphenyl)- $N$-(4-(pyridin-4-yl)thiazol-2-yl)-[1,2,4]triazolo[4,3-a]quinoxalin-4- amine(4t)}

light yellow solid, yield 83\%, m.p. 147-149 C, IR (KBr, v, $\mathrm{cm}^{-1}$ ): 3296 (N-H Str.), 3030 (Ar C-H Str.), ${ }^{1} \mathrm{H}$ NMR (400 MHz, DMSO- $\left.d_{6}\right) \delta_{\mathrm{H}}(\mathrm{ppm}): \delta 3.900\left(\mathrm{~s}, 3 \mathrm{H}, \mathrm{OCH}_{3}\right), 6.780-7.845(\mathrm{~m}, 13 \mathrm{H}, \mathrm{Ar}-$ $\mathrm{H}), 10.615(\mathrm{~s}, 1 \mathrm{H}, \mathrm{NH}) .{ }^{13} \mathrm{C} \mathrm{NMR}\left(100 \mathrm{MHz}, \mathrm{DMSO}-d_{6}\right) \delta_{\mathrm{C}}(\mathrm{ppm}): 57.20\left(\mathrm{OCH}_{3}\right), 112.22,113.50$, $114.18,115.23,116.20,118.70,120.12,122.23,124.13,125.19,126.08,127.55,128.88,129.15$, $130.19,131.20,133.66,134.10,136.10,138.10,140.22,142.82,143.20$ (Ar-C). MS(M $\left.{ }^{+}\right): 451.12$, Anal. Calcd. forC ${ }_{24} \mathrm{H}_{17} \mathrm{~N}_{7} \mathrm{OS}$ (451.50): C 63.84, $\mathrm{H}$ 3.80, N 21.72 Found: C 63.74, $\mathrm{H} 3.62, \mathrm{~N}$ $21.58 \%$. 


\section{CONCLUSION}

A series of some new $[1,2,4]$ triazolo[4,3-a]quinoxaline derivatives $\mathbf{4 a - 4 t}$ with pyridine and thiazole moiety has been synthesized via cyclisation reaction catalised by iodobenzene diacetate. This synthetic strategy allows the construction of relatively complicated triazolo quinoxaline frameworks equipped with a pyridine, thiazole unit and introduction of substituted phenyl ring on triazole moiety with fascinating yield in short time.

\section{Acknowledgments}

The authors are thankful to the Head, Department of Chemistry, Saurashtra University, Rajkot for providing ${ }^{1} \mathrm{H}$ NMR , ${ }^{13} \mathrm{C}$ NMR, mass and FT-IR spectroscopy and research facilities.

\section{References}

[1] Xuan X., Zhiguang X., Xiang L., Weihua L., J. Med. Chem. 11 (2001) 317.

[2] Zitouni G., Kaplancıklı Z. A., Yıldız M. T., Chevallet P., Eur. J. Med. Chem. 40 (2005) 607.

[3] Demirbas N., Karaoglu S. A., Demirbas A., Sanak K., Eur. J. Med. Chem. 39 (2004) 793.

[4] Kane J. M., Baron B. M., Dudley M. W., Sorensen S. M., Staeger M. A., Millar F. P., J. Med. Chem. 33 (1990) 2772.

[5] Holla B. S., Veerendra B., Shivananada M. K., Pujari B., Eur. J. Med. Chem. 28 (2003) 759.

[6] Tozkoparan B., Kupeli E., Yesilada E., Ertan M., Bioorg. Med. Chem. 15 (2007) 1808.

[7] Holla B. S., Veerendra B., Shivananada M. K., Pujari B., Eur. J. Med. Chem. 38 (2003) 759.

[8] Abdel-Aal M. T., El-Sayed W. A., El-Kosy S. M., El-Ashry E. S. H., Arch. Pharm. Chem. Life Sci. 341 (2008) 307.

[9] Chai B., Qian X., Cao S., Liu H., Song G., Arkivoc. 2 (2003) 141.

[10] Modzelewska-Banachiewicz B., Banachiewicz J., Chodkowska A., Jagiello-Wojtowicz E., Eur. J. Med. Chem. 39 ( 2004) 873.

[11] Jaso A., Zarranz B., Aldana I., Monge A., J. Med. Chem. 48 (2005) 2019.

[12] Sarges R., Howard H. R., Browne R. G., Lebel, L. A., Seymour P. A., Koe B. K., J. Med. Chem. 33 (1990) 2240.

[13] Dailey S., Feast J. W., Peace R. J., Sage I. C., Till S., Wood E. L., J. Mater. Chem. 11 (2001) 2238.

[14] Bayoumi A., Ghiaty A., El-Morsy A., Abul-Khair H., Hassan B., Bulletin of Faculty of Pharmacy, Cairo University. 50 (2012) 141.

[15] Chowdary K., et al., Indian Journal of Chemistry. 38B (1999) 1371.

[16] Antonino L., Guarcello A., Tetrahedron Letters. 49 (2008) 1847.

[17] Catarzi D., Colotta V., Varano F., J. Med. Chem. 48 (2005) 7932.

[18] Kondapalli V., Rao V., Kumar D., Bull. Korean Chem. Soc. 32 (2011) 8.

[19] Dodson R. M., King L. C., J Am Chem Soc. 67 (1945) 2242.

[20] Khunt H., Pipaliya P., Ghelani S., Naliapara Y. T., International Letters of Chemistry, Physics and Astronomy 24 (2014) 134.

[21] Patel A. S., Khunt H., Babariya J., Ghelani S., Naliapara Y. T., International Letters of Chemistry, Physics and Astronomy 30 (2014) 106. 
[22] Gami P., Vilapara K., Khunt H., Babariya J., Naliapara Y. T., International Letters of Chemistry, Physics and Astronomy 30 (2014) 127.

[23] Khunt H., Babariya J., Dedakiya C., Naliapara Y. T., International Letters of Chemistry, Physics and Astronomy 30 (2014) 185.

[24] Khunt H., Babariya J., Naliapara Y. T., International Letters of Chemistry, Physics and Astronomy 31 (2014) 20.

[25] Prajapati D., Vilapara K., Naliapara Y. T., International Letters of Chemistry, Physics and Astronomy 33 (2014) 12. 